\title{
Investigation of potential DNA damaging and apoptotic effects of PLK1 inhibitor SBE13 in breast cancer cell line MDA-MB-231
}

\author{
Mustafa ERGUL ${ }^{1, *(1)}$

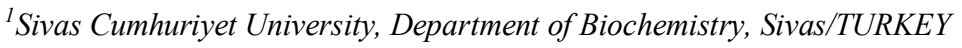

\begin{abstract}
Polo-like kinase 1 (PLK1) regulates various steps of mitosis and aberrantly expressed in several tumor types. As elevated levels of PLK1 contributes to tumorigenesis and poor prognosis, specific inhibition of PLK1 has garnered increasing attention in recent years in anticancer studies. The objective of this study was to examine cytotoxic, apoptotic, and DNA-damaging potentials of SBE13, a PLK1 inhibitor, against MDA-MB-231 breast cancer cells. The regulatory efficacy of SBE13 on cell cycle arrest was also determined. Cytotoxicity of SBE13 was assessed via XTT assay. Apoptosis, cell cycle distribution, and DNA damage response were also examined using the flow cytometry assay. The results revealed that SBE13 had a dose-dependent cytotoxic effect in MDA-MB-231 cells. This compound has also induced cell cycle arrest at the $\mathrm{G} 2 / \mathrm{M}$ point and significantly promoted apoptosis and DNA damage response in MDA-MB-231 cells. Collectively, these data pointed out that SBE13 can be regarded as a suitable candidate for the therapy of breast cancer. However, further studies are required to consolidate the anticancer activity of SBE13.
\end{abstract}

\section{Article info}

History:

Received: 27.07 .2020

Accepted: 22.11 .2020

Keywords:

PLK1 inhibition,

SBE13,

Breast Cancer,

Apoptosis,

DNA damage.

\section{Introduction}

Cancer remains the second leading cause of death. Despite new advancements in its diagnosis and management, it contributes to important morbidities and mortality in patients [1]. Although there are many treatment modalities in cancer therapy, chemotherapy remains to be a popular treatment strategy. However, it is often related to significant adverse effects; therefore, the development of effective and especially targetedanticancer agents is an important prerequisite for successful and satisfactory chemotherapy [2]. Globally, breast cancer the most frequently diagnosed malignancy in the woman. Despite recently introduced chemotherapeutic agents, resistance development of breast cancer cells continues to be an important contributor to treatment failure. Thus, more effective and novel strategies considering different signal transduction pathways to improve the success of chemotherapy and to reduce drug resistance are required for breast cancer treatment [3].
Polo-like kinases (PLKs) are ser/thr kinases playing critical roles in mitosis and DNA damage response. In humans, PLKs consist of five isoforms, among these isoforms, PLK1 is the best characterized and the most widely studied in cancer pathogenesis. Normally, PLK1 is moderately expressed in normal cells during mitosis and plays a vital role in the mitotic processes such as spindle formation, centrosome maturation/separation, chromatid separation, and cytokinesis execution [2, 4]. Moreover, its overexpression has been detected in a wide range of cancers such as head and neck, non-small cell lung, and esophageal cancers [5]. Additionally, increased expression of PLK1 has been strongly correlated with worse prognosis and thus, PLK1 seems to be considered as a suitable target for chemotherapeutic agents.

To date, several PLK1 inhibitors such as GSK461364, BI 2536, and RO3280 have been developed and investigated as potential anticancer drugs and they have shown promising results in cancer cells. Many studies have also presented that depletion of PLK1 causes decreased cell viability, DNA damage response, cell cycle arrest, and finally apoptosis in tumor cells [6, 7, 8]. One of these inhibitors is SBE13 and this PLK1specific inhibitor demonstrated anticancer effects in 
several tumor cells with EC50 values ranging from 5 $\mu \mathrm{M}$ to $39 \mu \mathrm{M}$ and caused apoptosis [9].

In the light of the above-mentioned observations regarding PLK1 inhibition, the current study was conducted to explore the cytotoxic actions of SBE13 against breast cancer cell line MDA-MB-231. Although it is known that SBE13 exhibits an anticancer effect on various human cancer cell types, the anticancer potential of this compound in many tumor cells including MDA-MB-231 has not been elucidated yet. Therefore, in this work, SBE13 was selected as a study compound.

\section{Materials and Methods}

\subsection{Cell culture}

Human breast cancer cell line MDA-MB-231 (ATCC, HTB-26) was obtained from ATCC and cultured in DMEM (Gibco Thermo Fisher Scientific) supplemented with $10 \%$ fetal bovine serum (SigmaAldrich) and $50 \mathrm{U} / \mathrm{mL}$ penicillin/streptomycin (SigmaAldrich). The cells were incubated at $37^{\circ} \mathrm{C}$ within a $5 \%$ $\mathrm{CO}_{2}$ humidified atmosphere.

\subsection{Cell viability assay}

The stock solution of SBE13 (Cayman) was prepared in DMSO at a final concentration of $10 \mathrm{mM}$. Cell viability was examined by a standard XTT assay (Roche Diagnostic) after $48 \mathrm{~h}$ incubation as described previously [2]. Briefly, cells were treated with various concentrations $(1,2.5,5,10,20$, and $40 \mu \mathrm{M})$ for $48 \mathrm{~h}$. Then XTT assay was performed. All the experiments were done in triplicate and the cell viability was evaluated as viable cell amount percent compared to control cells. The half maximal inhibitory concentration $\left(\mathrm{IC}_{50}\right)$ value of SBE13 in MDA-MB-231 cells was determined using the Graph Prism 7 software.

\subsection{Cell cycle analysis}

We assessed the cell cycle distribution by Muse Cell Cycle Assay Kit (Merck Millipore) according to the guideline of the producer. Before incubating for attachment, $5 \times 10^{5}$ MDA-MB-231 cells were plated into six-well plates. After confirmation of attachment, the cells were exposed to SBE13 at $\mathrm{IC}_{50}$ concentration and incubated for $48 \mathrm{~h}$. The cells were then washed with phosphate-buffered saline (PBS) and fixed in 1 $\mathrm{mL}$ cold $70 \%(\mathrm{v} / \mathrm{v})$ ethanol. Later, the fixed cells were washed once with PBS, and incubated with $200 \mu \mathrm{L}$ of assay solution for $30 \mathrm{~min}$ in the dark at room temperature. Finally, different cell cycle stage (G0/G1, $\mathrm{S}$, and $\mathrm{G} 2 / \mathrm{M}$ ) percentages of the cells were calculated by Muse Cell Analyzer (Millipore).

\subsection{Annexin $\mathrm{V}$ binding assay}

Initially, MDA-MB-231 cells were plated into six-well plates and incubated for attachment. The cells then were exposed to SBE13 at $\mathrm{IC}_{50}$ concentration and incubated for $48 \mathrm{~h}$. The cells were harvested, incubated with Muse ${ }^{\mathrm{TM}}$ Annexin V \& Dead Cell kit reagent, and the manufacturer's protocol were followed. Four different populations were monitored by using Annexin V and/or 7-AAD positivity on Cell Analyzer (Muse, Millipore): Live, early apoptotic, late apoptotic, and dead.

\subsection{DNA damage assay}

MDA-MB-231 cells were exposed to SBE13 at the $\mathrm{IC}_{50}$ concentration for $48 \mathrm{~h}$ and the induction of ATM and H2AX was assessed by Muse Multi-Color DNA Damage kit (Merck Millipore) according to the report in our previous study [2].

\subsection{Statistical analysis}

Data obtained from the experiments were expressed as the mean \pm standard deviation. Kruskal-Wallis ANOVA test with post hoc Dunn's test or MannWhitney test were used as appropriate to compare the variables measured after SBE13 exposition versus controls. Targeting of PLK1 activity through SBE13 counteracts cell viability of MDA-MB-231 cells

\section{Results}

\subsection{Targeting of PLK1 activity through SBE13 counteracts cell viability of MDA-MB-231 cells}

The cytotoxicity of the PLK1 inhibitor SBE13 has been evaluated in MDA-MB-231 cells. The cells were exposed to diverse concentrations of SBE13 $(0-40 \mu \mathrm{M})$ for $48 \mathrm{~h}$ and its antiproliferative effect was evaluated using the XTT assay. Cell viability results are represented in Figure 1 and it has been shown that SBE13 has a dose-dependent inhibitory effect at $48 \mathrm{~h}$ when compared to the untreated cells $(\mathrm{P}<0.01)$. 


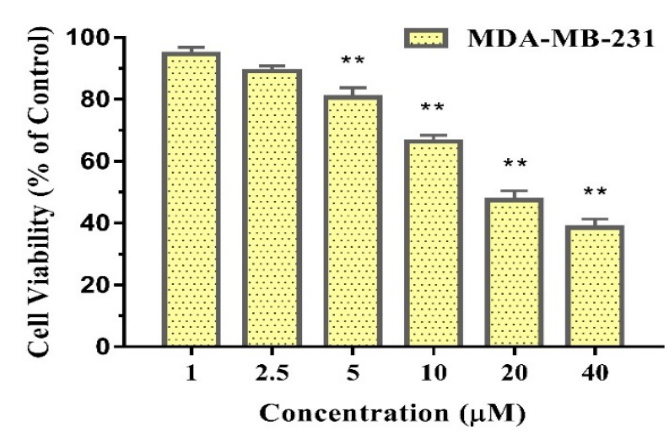

Figure 1. Cell viability percentages of MDA-MB-231 cells were treated with SBE13 at concentrations from 1 to $40 \mu \mathrm{M}$ for $48 \mathrm{~h}$, measured with XTT assay. Data are presented as mean with a standard deviation of the three replicates. **Significantly different when compared to cell viability of control cells $(\mathrm{P}<0.01)$.

The $\mathrm{IC}_{50}$ value of SBE13 was recorded as $15.7 \mu \mathrm{M}$ for $48 \mathrm{~h}$ in MDA-MB-231 cells and this concentration was used for the subsequent experiments.

\subsection{SBE13 induces cell cycle arrest in MDA-MB- 231 cells}

Next, to determine the cell cycle distribution effect of SBE13 in MDA-MB-231 cells, cell cycle assay was done and the results are displayed in Figure 2.

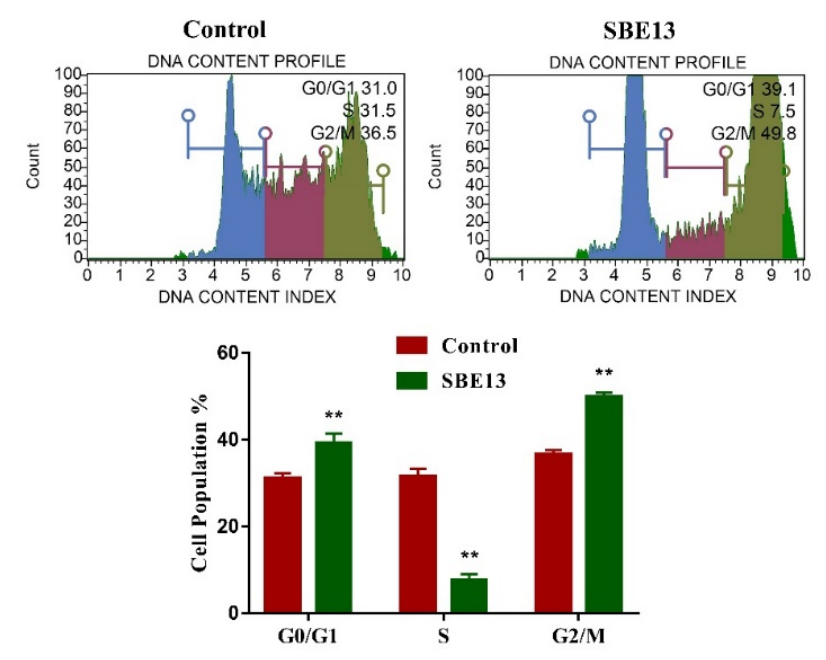

Figure 2. Regulatory effect of SBE13 on cell cycle progression. The MDA-MB-231 cells were treated with SBE13 with $\mathrm{IC}_{50}$ value of $15.7 \mu \mathrm{M}$ for $48 \mathrm{~h}$ and the cell population rates are presented as mean with a standard deviation of three replicates. ${ }^{*}$ Significantly different when compared to cell population rate of untreated cells $(\mathrm{P}<0.01)$.

The MDA-MB-231 cells were exposed to $15.7 \mu \mathrm{M}$ of SBE13 and the results indicated a significant increase in the percentage of the $\mathrm{G} 2 / \mathrm{M}$ cell by $49.8 \pm 1.10 \%$ in the SBE13 treated group when compared with untreated control $(36.5 \pm 1.12 \%)(\mathrm{P}<0.01)$. Moreover, while SBE13 treatment increased the G0/G1 population, it decreased the $\mathrm{S}$ population $(\mathrm{P}<0.01)$ as presented in Figure 2.

\subsection{SBE13 induces apoptosis in MDA-MB-231 cells}

The effect of SBE13 on the induction of apoptosis in MDA-MB-231 cells was also assessed by the Annexin $\mathrm{V}$ binding analyses via flow cytometry. According to the results, SBE13 treatment significantly promoted the early and late apoptotic cell population when compared to the control cells $(\mathrm{P}<0.01)$.

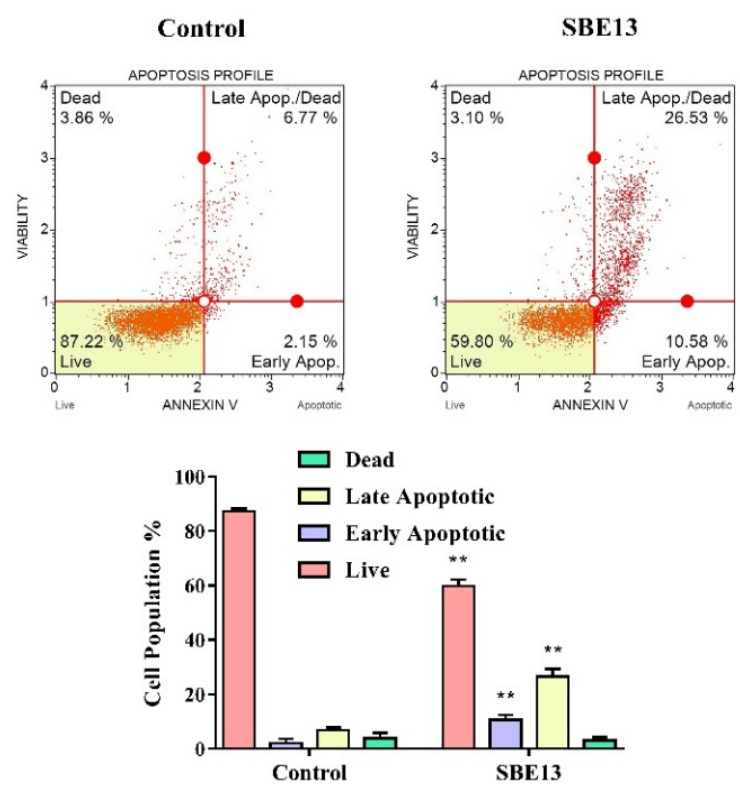

Figure 3. Cell population rate of MDA-MB-231 cells presenting apoptotic effects of SBE13 after exposed to the $\mathrm{IC}_{50}$ value of SBE13, determined with Annexin V assay. All experiments were performed as at least three replicates. **Significantly different when compared to cell population rate of untreated cells $(\mathrm{P}<0.01)$.

As visible in Figure 3, the early and late apoptotic cell population $\%$ in untreated cells $(2.15 \pm 1.46 \%$, $6.77 \pm 1.01 \%$, respectively) significantly increased to $10.58 \pm 1.79 \%$ and $26.53 \pm 2$. $75 \%$, respectively, in SBE13 administered group $(\mathrm{P}<0.01)$.

\subsection{SBE13 induces DNA damage response in MDA- MB-231 cells}

Subsequently, to clarify SBE13 treatment on DNA damage response in MDA-MB-231 cells, the cells were treated with $\mathrm{IC}_{50}$ concentration of SBE13 for 48 $\mathrm{h}$, and then the Muse DNA damage assay was performed. According to the results SBE13 treatment 
markedly induced DNA damage response in MDAMB-231 cells when compared to untreated cells.
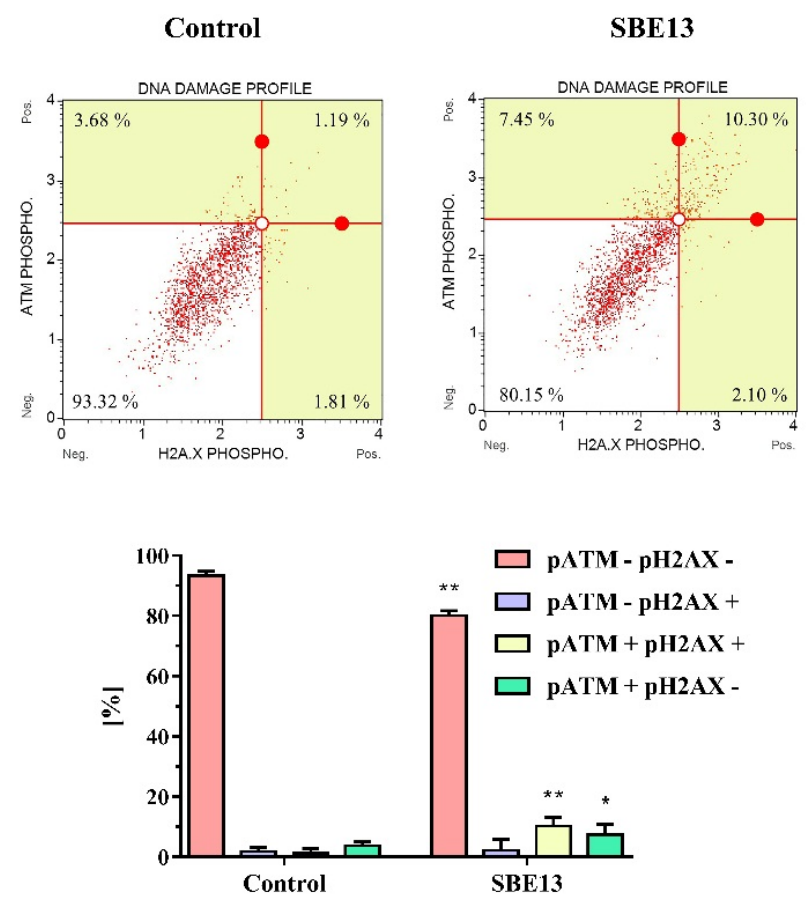

Figure 4. DNA damage response rates of MDA-MB231cells administered SBE13 with $\mathrm{IC}_{50}$ concentration for $48 \mathrm{~h}$, determined with activation of ATM and H2AX. Data are expressed as mean with standard deviation, ${ }^{*} \mathrm{P}$ $<0.05,{ }^{* *} \mathrm{P}<0.01$.

Figure 4 showed that percentage of DNA doublestrand breaks (pATM $+\mathrm{pH} 2 \mathrm{AX}+$ ) and single-strand breaks $(\mathrm{pATM}+\mathrm{pH} 2 \mathrm{AX}-)$ in untreated cells $(1.19 \pm 1.50 \%, 3.68 \% \pm 1.27$, respectively) increased to $10.30 \pm 2.71 \%$ and $7.45 \% \pm 3.20$, respectively, in SBE13 treated group $(\mathrm{P}<0.01)$. DNA damage data also suggested that SBE13 possess a genotoxic efficacy on MDA-MB-231 cells.

\section{Discussion}

In the present study, a series of experiments were performed to evaluate the cytotoxic and apoptotic effects of SBE13 on MDA-MB-231 cells representing breast cancer. Furthermore, the DNA damage response of SBE13 was examined. The cytotoxicity experiments showed that SBE13 treatment significantly suppressed cell viability. Flow cytometry studies also revealed that SBE13 treatment induced cell cycle arrest at the G2/M phase, induced apoptosis, and caused DNA damage to MDA-MB-231 cells. For the first time, overall, these findings suggest that SBE13 has significant anticancer effects in MDA-MB-231 cells.
In many cancer cell types, pharmacologic perturbation of PLK1 activity via selective inhibitors induces cell death through apoptosis and this makes PLK1 a fascinating anticancer drug target. In this context, numerous studies have reported the anticancer effects of PLK1 inhibition in solid tumors and hematologic malignancies. Keppner and coworkers [10] analyzed anticancer effects of SBE13 in different cancer cell lines and according to their results, SBE13 exhibited cytotoxic effect in a dose-depended manner in HCT15, MCF-7, BT-474, A431, SK-OV-3, and HeLa cancer cell lines. Additionally, particularly high concentrations of SBE13 caused cell cycle arrest at the G2/M point and ultimately led to apoptosis. SBE13 treatment also caused abnormal mitotic figures in HeLa cell lines. Similarly, it has been demonstrated that SBE13 treatment significantly reduced the cell viability of HeLa cells [11]. In another study, Lange and colleagues [12] explored whether SBE13 and PCK $\beta$ inhibitor enzastaurin acted synergistically to suppress the growth of diverse tumor cells such as HeLa, MCF-7, hTERT-RPE1, HCT116p53wt, and HCT116p53-/-. In that study, SBE13 and enzastaurin showed synergistic effects on the cancer cell lines excluding hTERT-RPE1. Furthermore, it has been revealed that combination of SBE13 and enzastaurin enhances the apoptosis in HeLa cells when compared to enzastaurin alone.

In the literature, many studies examined the anticancer efficacy of various PLK1 inhibitors such as BI 6727, RO3280, BI2536, and GSK461364A [13]. In our previous study [2], we have revealed that RO3280 has significant anticancer effects on breast cancer cell line MCF-7 and that RO3280 suppresses cell proliferation through arresting cell cycle at G2/M point and causes apoptosis by inducing total caspase levels and annexin $\mathrm{V}$ binding. We also exhibited that RO3280 treatment significantly decreases mitochondrial membrane potential and increases DNA damage. Similarly, in the current research on MDA-MB-231 cells, SBE13 displayed a significant anticancer effect in manners of stimulating cell cycle arrest at G2/M point, inducing DNA damage, and leading the cells to apoptosis. In a similar fashion, it has been exhibited that depletion of PLK1 activity through BI 2536 reduced proliferation of A549, A427, and NCIH1299 cells. BI 2536 also caused cell cycle arrest at the G2/M phase and disorientation of centrosomes and mitotic spindles in non-small cell lung cancer cells [8]. In a different study on the same inhibitor, Wu et al. [4] examined whether BI 2536 and cisplatin acted synergistically to inhibit the growth of esophageal squamous cell carcinoma (ESCC). Their results showed that combination of BI2536 and cisplatin synergistically killed ESCC cells. 
Their further studies also exhibited that BI2536 treatment caused significant DNA damage and disrupted the DNA damage repair in cisplatin-treated cells both in vitro and in vivo. These results and our findings showed that PLK1 inhibition causes significant DNA damage. It has also been shown that inhibition of PLK1 through BI6727 caused cell cycle G2-phase arrest, induced apoptosis, and inhibited migration of gastric cancer cells [14]. Another PLK1 inhibitor, TAK-960, was tested against multiple solid and hematologic malignancies such as A2780, SW620, HT-29, K562 and it was showed potent antiproliferative activity in all tested cancer cell lines. TAK-960 treatment also induced cell cycle arrest at the G2/M phase and significantly suppressed the growth of HT-29 colorectal cancer xenografts [15]. Recently, other researchers examined the anticancer potency of PLK1 inhibitor GSK461364A on glioblastoma multiforme cell lines and found that PLK1 inhibition arrested cells at the G2/M phase and caused significant DNA damage. Their in vivo results also revealed that PLK1 inhibition significantly reduced tumor volume and enhance radiation sensitivity in glioblastoma multiforme [16].

\section{Conclusion}

Taken together, all the reports mentioned above demonstrate that PLK1 plays a significant role in the development and progress of human cancers and its inhibition is specifically associated with reduced proliferation of cancer cells. In accordance with the findings of studies examined PLK1 inhibitors in several cancer cell lines, SBE13, another PLK1 inhibitor, seems to have meaningful cytotoxic activity via cell cycle arrest in breast cancer cell line MDAMB-231. SBE13 also provides DNA damage and apoptosis in MDA-MB-231 cells. SBE13 may be considered a promising anticancer agent for the treatment of breast cancer. However, there is a need to conduct further in vitro and in vivo studies to elucidate the potential use of SBE13 for breast cancer treatment.

\section{Acknowledgement}

This work was supported by Cumhuriyet University Scientific Research Project (CUBAP, Project Number: ECZ-045). The author would like to thank the CUTFAM Research Center, for providing the necessary facilities to conduct this study.

\section{Conflicts of interest}

The author declared no conflict of interest. 


\section{References}

[1] Siegel R.L., Miller K.D., Jemal A. Cancer statistics, 2019, CA Cancer J Clin., 69(1) (2019) 7-34.

[2] Ergul M., Bakar-Ates F. RO3280: A Novel PLK1 Inhibitor, Suppressed the Proliferation of MCF-7 Breast Cancer Cells Through the Induction of Cell Cycle Arrest at G2/M Point, Anticancer Agents Med Chem., 19(15) (2019) 1846-1854.

[3] Howell A., Anderson A.S., Clarke R.B., Duffy S.W., Evans D.G., Garcia-Closas M., Gescher A.J., Key T.J., Saxton J.M., Harvie, M.N. Risk determination and prevention of breast cancer, Breast cancer research: BCR, 16(5) (2014) 446.

[4] Wu M., Wang Y., Yang D., Gong Y., Rao F., Liu R., Danna Y., Li J., Fan J., Chen J., Zhang W., Zhan, Q.A PLK1 kinase inhibitor enhances the chemosensitivity of cisplatin by inducing pyroptosis in oesophageal squamous cell carcinoma, EBioMedicine, 41 (2019) 244-255.

[5] de Cárcer G., Venkateswaran S. V., Salgueiro L., El Bakkali A., Somogyi K., Rowald K., Montañés, P., Sanclemente M., Escobar B., de Martino A., McGranahan N., Malumbres M., Sotillo, R. Plk1 overexpression induces chromosomal instability and suppresses tumor development, Nature communications, 9(1) (2018) 3012.

[6] Smith L., Farzan R., Ali S., Buluwela L., Saurin A. T., \& Meek D.W. The responses of cancer cells to PLK1 inhibitors reveal a novel protective role for p53 in maintaining centrosome separation, Scientific reports, 7(1) (2017) 16115.

[7] Wang N.N., Li Z.H., Zhao H., Tao Y. F., Xu L. X., Lu J., Cao L., Du X. J., Sun L. C., Zhao W. L., Xiao P. F., Fang F., Su G. H., Li Y. H., Li G., Li Y. P., Xu Y. Y., Zhou H. T., Wu Y., Jin M. F., Pan J. Molecular targeting of the oncoprotein PLK1 in pediatric acute myeloid leukemia: RO3280, a novel PLK1 inhibitor, induces apoptosis in leukemia cells, International journal of molecular sciences, 16(1) (2015) 1266-1292.

[8] Choi M., Kim W., Cheon M. G., Lee C. W., \& Kim J.E. Polo-like kinase 1 inhibitor BI2536 causes mitotic catastrophe following activation of the spindle assembly checkpoint in non-small cell lung cancer cells, Cancer letters, 357(2) (2015) 591-601.
[9] Keppner S., Proschak E., Schneider G., Spänkuch B. SBE13, a newly identified inhibitor of inactive polo-like kinase 1, J Cheminform. (2010) 2(Suppl 1) P54.

[10] Keppner S., Proschak E., Kaufmann M., Strebhardt K., Schneider G., \& Spänkuch B. Biological impact of freezing Plk1 in its inactive conformation in cancer cells, Cell cycle, 9(4) (2010) 761-773.

[11] Keppner S., Proschak E., Schneider G., \& Spänkuch B. Identification and validation of a potent type II inhibitor of inactive polo-like kinase 1, ChemMedChem, 4(11) (2009) 1806-1809.

[12] Lange L., Keppner-Witter S., Grigat J., \& Spänkuch B. Combinatorial inhibition of Plk1 and $\mathrm{PKC} \beta$ in cancer cells with different p53 status, Oncotarget, 5(8) (2014) 2263-2275.

[13] Gutteridge R. E., Ndiaye M. A., Liu X., \& Ahmad N. Plk1 Inhibitors in Cancer Therapy: From Laboratory to Clinics, Molecular cancer therapeutics, 15(7) (2016) 1427-1435.

[14] Dang S.C., Fan Y.Y., Cui L., Chen J.X., Qu J.G., $\mathrm{Gu}$ M. PLK1 as a potential prognostic marker of gastric cancer through MEK-ERK pathway on PDTX models, OncoTargets and therapy, 11 (2018) 6239-6247.

[15] Hikichi Y., Honda K., Hikami K., Miyashita H., Kaieda I., Murai S., Uchiyama N., Hasegawa M., Kawamoto T., Sato T., Ichikawa T., Cao S., Nie Z., Zhang L., Yang J., Kuida K., \& Kupperman E. TAK-960, a novel, orally available, selective inhibitor of polo-like kinase 1, shows broadspectrum preclinical antitumor activity in multiple dosing regimens, Molecular cancer therapeutics, 11(3) (2012) 700-709.

[16] Tandle A.T., Kramp T., Kil W. J., Halthore A., Gehlhaus K., Shankavaram U., Tofilon P.J., Caplen N.J., \& Camphausen K. Inhibition of pololike kinase 1 in glioblastoma multiforme induces mitotic catastrophe and enhances radiosensitisation, Albayrak G., Korkmaz F.D., Bali E.B., Bagriacik E.U., Antitumorigenic Effect of Memantine via Interfering Glutamate Metabolism in Mouse 4T1 Breast Tumor Model, Anticancer Agents Med Chem., (2020 European journal of cancer, 49(14) (2013) 3020-3028. 